\title{
Sobre los teoremas de rotura, sus corolarios y su aplicación al cálculo de arcos de fábrica
}

\author{
On limit analysis theorems, their corollaries \\ and their appliance to masonry arches analysis
}

$\underline{\text { V. Quintas }}^{(*)}$

\section{RESUMEN}

En este trabajo se enuncian los teoremas de rotura y sus corolarios para aplicarlos al cálculo en rotura de arcos. Después de analizar la teoría de arcos, se estudia el significado de la línea de presiones y de la línea de empujes desde el punto de vista del cálculo a rotura.

\section{$425-2$}

Palabras clave: arcos, fábrica, teorema de rotura.

\section{INTRODUCCIÓN}

Como cualquier persona dedicada a la docencia de estructuras sabe, el cálculo a rotura, a pesar de su aparente sencillez, es mucho más difícil de comprender que el cálculo elástico tradicional. Una de las razones de esta aparente paradoja es que su base, los teoremas de rotura, inducen a confusión, incluso, como veremos, con sus títulos. Esto explicaría que una de sus aplicaciones más populares, la utilización de la línea de empujes para el cálculo de arcos, se utilice sin conocer bien los parámetros de seguridad que se manejan.

Para poder estudiar esto en detalle vamos a examinar en primer lugar los teoremas de rotura, y una vez repasada la estática de los arcos, aplicarlos a los arcos de fábrica.

\section{EL PRIMER TEOREMA DE ROTURA O DEL "LÍMITE SUPERIOR"}

El primer teorema de rotura trata de las configuraciones de rotura de una estructura

\section{SUMMARY}

In this work limit analysis theorems are exposed in order to study their appliance to the analysis of masonry arches. All this allows studying the role of lines of thrust and curves of pressure on the safety of arches.

Keywords: arches, masonry, analysis theorem.

que siendo "correctas", -es decir, transforman la estructura en un mecanismo, los esfuerzos últimos en las rótulas plásticas están en equilibrio con las cargas y además existe coherencia entre la forma de deformarse las rótulas y sus esfuerzos últimos y cumplen las condiciones estáticas de borde- no son la configuración real de colapso. Podría enunciarse como:

"A cualquier configuración de rotura correcta pero no real, le corresponde una carga de rotura mayor que la real". Evidentemente, si encontrásemos una configuración de rotura correcta con una carga de rotura menor, la estructura rompería antes por esa configuración y pasaría a ser la configuración real. Dicho de otra forma, la carga de rotura es el límite inferior de todas las cargas de rotura posibles en configuraciones de colapso correctas. Como decíamos, el propio título del teorema: "del límite superior", induce a confusión. Su posible pervivencia puede deberse a su corolario, que es realmente el que utilizamos en el cálculo: "Si a las configuraciones de rotura correctas pero no reales les 
aplicamos la carga de rotura, los esfuerzos últimos que aparecen son siempre menores que los esfuerzos últimos de la configuración real de rotura". Obviamente, en la configuración real la carga de rotura es menor porque aparecen esfuerzos mayores, y en este caso, el esfuerzo último de rotura sí es el límite superior de todos los esfuerzos últimos de las configuraciones de rotura no reales. Johansen (1) enunció la aplicación de este corolario a placas como su "V Teorema": "La configuración de rotura real corresponde al máximo valor del momento último por unidad de longitud". Realmente este corolario es la base de lo que podríamos llamar en sentido estricto "Método de cálculo en rotura". Una vez definida la carga de rotura se buscan todas las configuraciones de rotura posibles y correctas, se obtienen sus esfuerzos - por equilibrio o expresando el trabajo virtual del mecanismo- se elige la que tenga el máximo esfuerzo último, y se dimensiona la estructura para que el esfuerzo último de la sección tenga el mismo valor que el esfuerzo último de la estructura con la carga de colapso. Este proceso en algunos casos es sencillísimo, en otros -como el de algunas placas- puede llegar a ser extremadamente complicado.

El significado profundo de este Teorema es que las configuraciones de rotura correctas pero no reales, no cumplen la condición de "cedencia" (2), es decir, en zonas de la estructura que no son las rótulas plásticas existe un esfuerzo mayor que el que existe en las rótulas y que hemos supuesto que es el esfuerzo último.
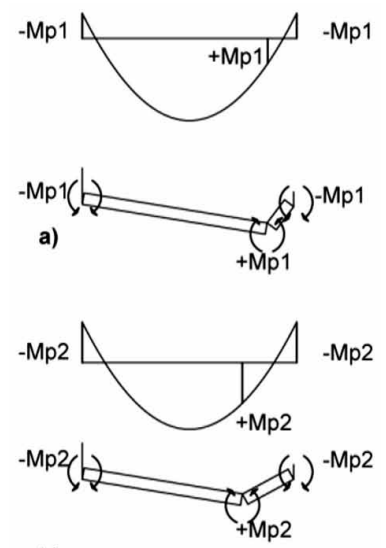

b)

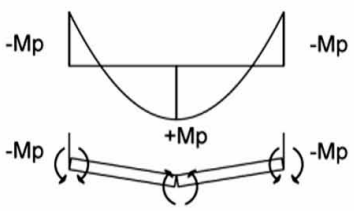

c)

$+\mathrm{Mp}$

1. Aplicación del primer teorema una viga empotrada con carga uniforme. borde y un tipo de carga determinados sólo existe una configuración y una carga de rotura". Este teorema parece simplemente obvio: cualquier fenómeno mecánico -la trayectoria de un proyectil o el movimiento de un péndulo- tiene sólo una solución para unas condiciones dadas, salvo que estemos en régimen caótico. Sin embargo el significado de este Teorema es más profundo: la configuración y la carga de rotura no depende del estado en que se encuentra la estructura en servicio: momentos elásticos o auto-tensiones debidas, por ejemplo, a un pretensado o un movimiento en los apoyos.

Para comprenderlo (Figura 2) podemos volver al ejemplo de la viga empotrada de la Figura 1, pero en este caso con distintas condiciones de empotramiento elásticas: en a) está limitada por dos vanos de la misma sección uno, corto, con carga, y otro, largo, descargado; en b) por dos vanos descargados y en c) la viga se dobla formando un pórtico de dos articulaciones. Los momentos elásticos de las tres situaciones son muy distintos uno del otro, sin embargo la rotura es idéntica en los tres casos: es la de la Figura 1. Efectivamente si, por ejemplo, imaginamos el proceso de rotura de la estructura a) veremos (Figura 3) que si empezamos a aumentar la carga, habrá un valor de ésta para la que en el apoyo derecho se alcance el momento último de la sección $\mathrm{Mp}$, con lo que se formará una rótula plástica (Figura 3.1) y al continuar aumentando la carga se bloqueará el momento en este punto. Cuando en el vano se alcance el valor de Mp, se formará otra rótula y se bloqueará el valor del momento en ese punto (Figura 3.2). Finalmente, si continuamos aumentando la carga, ésta alcanzará el valor de rotura cuando en el apoyo izquierdo el momento valga $\mathrm{Mp}$ y se forme un mecanismo con una carga de rotura (Figura 3.3) idénticos a los de la Figura 1. Lo mismo podría deducirse estudiando el proceso de rotura de las estructuras b) y c), el orden de la aparición de las rótulas puede ser distinto, pero el resultado final es siempre el mismo.

La configuración y la carga de rotura dependen de las condiciones de borde estáticas -en este caso dos empotramientos- pero nunca de las condiciones de borde elásticas.

Ya veremos la importancia que tiene este teorema para el cálculo de arcos. El teorema tiene como limitación que el material sea perfectamente plástico: si el proceso que hemos descrito implica alargamientos en las fibras que produzcan la rotura del material, la estructura puede romper antes de alcanzar la configuración de colapso definida por sus condiciones de borde estáticas. Ésta fue 
la razón de la llamada "paradoja de las rótulas plásticas" (3): una viga como la b) de la Figura 2, rompía antes de lo previsto debido a que para alcanzar la configuración de rotura debía hacer giros excesivos, y es también la razón por la que es tan difícil aplicar el cálculo de rotura a las vigas de hormigón armado.

Aparentemente, sin embargo, todas estas incertidumbres pueden resolverse si aplicamos el segundo teorema de rotura o del "límite inferior", que estudiamos a continuación.

\section{EL SEGUNDO TEOREMA DE ROTURA O DEL" LÍMITE INFERIOR"}

El segundo teorema trata de configuraciones de equilibrio no de rotura de la estructura, es decir, de un régimen de tensiones o esfuerzos dentro de la estructura que cumpla las condiciones de contorno, pero que no suponga que la estructura se transforme en un mecanismo. El mejor enunciado es el de Nielsen (4), que se puede escribir, algo modificado, como: "Si para la carga supuesta de rotura podemos encontrar dentro de la estructura una configuración de equilibrio no de rotura que la resista, la carga real de rotura debe ser mayor que la supuesta". No es de extrañar (3) que uno de sus primeros enunciados se hiciera sin demostración: si la estructura es capaz de resistir la supuesta carga de rotura con una configuración de equilibrio sin transformarse en un mecanismo, tiene las suficientes reservas de resistencia como para que se pueda afirmar que la carga de rotura, es decir, la carga que la transforma en un mecanismo, es mayor. Este teorema es por supuesto extraordinariamente útil para comprobar si estructuras existentes -como las históricas- están sobredimensionadas, sin más que comprobar si una configuración de equilibrio dentro de la estructura resiste la carga que suponemos debería hacer colapsar la estructura si estuviese estrictamente dimensionada.

Quizá por hacer un enunciado simétrico al del primer teorema se le suele expresar como: "Las configuraciones de equilibrio no de rotura que se pueden encontrar dentro de la estructura corresponden a estructuras cuya carga de rotura es menor". Así (Figura 4) si volvemos al ejemplo de una viga empotrada de sección constante cuyo momento último es $\mathrm{Mp}$, si comprobamos que para la carga última la viga es capaz de resistir los diagramas de momentos -es decir, las "configuraciones de equilibrio"- a) y b) sin romperse, podemos afirmar que su carga de rotura es mayor que la supuesta y mayor que las que producirían la rotura de las estructuras correspondientes a estos diagramas, es decir, las estructuras acarteladas sombrea- das que se han dibujado dentro de la viga, que serían las que colapsarían con estos diagramas.

Como ocurría con el primer teorema, su título "del límite inferior", induce a confusión, porque lo que realmente dice el teorema es que la carga de rotura real es el límite superior de las cargas de rotura correspondientes a las configuraciones de equilibrio. Como antes, su corolario, vuelve a hacer correcto este título. Podemos enunciarlo como: "Si a cualquier configuración de equilibrio no de rotura que encontremos dentro de la estructura le aplicamos la carga de rotura, su esfuerzo máximo será mayor que los esfuerzos últimos de la estructura". Evidentemente, si suponemos que en los diagramas de la Figura 4 hemos aplicado la carga de rotura, sus valores máximos son mayores que el momento último de la estructura que corresponde al diagrama de la Figura 1, y por tanto el momento último es el límite inferior de los momentos máximos de las configuraciones de equilibrio, si se les aplica la carga de rotura.

Es importante subrayar el término "esfuerzo máximo", porque-como se puede ver en los diagramas de la Figura 4- los otros máximos de esfuerzos pueden ser menores $-y$ de hecho casi siempre lo son- que los esfuerzos de rotura en esos puntos, incluso que los esfuerzos en servicio en algunos casos. El segundo teorema puede ser un buen método para comprobar si la estructura está sobredimensionada, pero de ninguna manera puede tomársele como base de un método de diseño. En tal caso el llamado "teorema de la seguridad" se transforma peligrosamente en el "teorema de la inseguridad".

Por supuesto, como dice Schlaigh (5), si armamos una viga de hormigón armado, perfectamente empotrada por ejemplo, a partir del diagrama a) de la Figura 4, la estructura se "acomodará", hasta que su diagrama en rotura coincida con el del diseño, pero a costa de fisuraciones, giros y alargamientos del material que pueden llegar a ser inadmisibles.

La aplicación del segundo teorema a placas ha producido todo un género de métodos de cálculo de placas: son las soluciones de "límite inferior" (4). Sencillamente, se busca una solución polinómica para los esfuerzos que cumpla las condiciones de equilibrio en todos los puntos y las condiciones estáticas del contorno, y se iguala su máximo momento para la carga última al momento último de la sección, sabiendo que va a ser mayor que el de rotura. Una variante es el "método de las bandas" en el que se supone que la placa no tiene resistencia a momentos

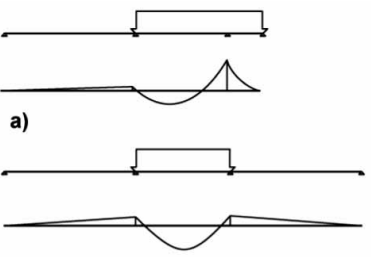

b)
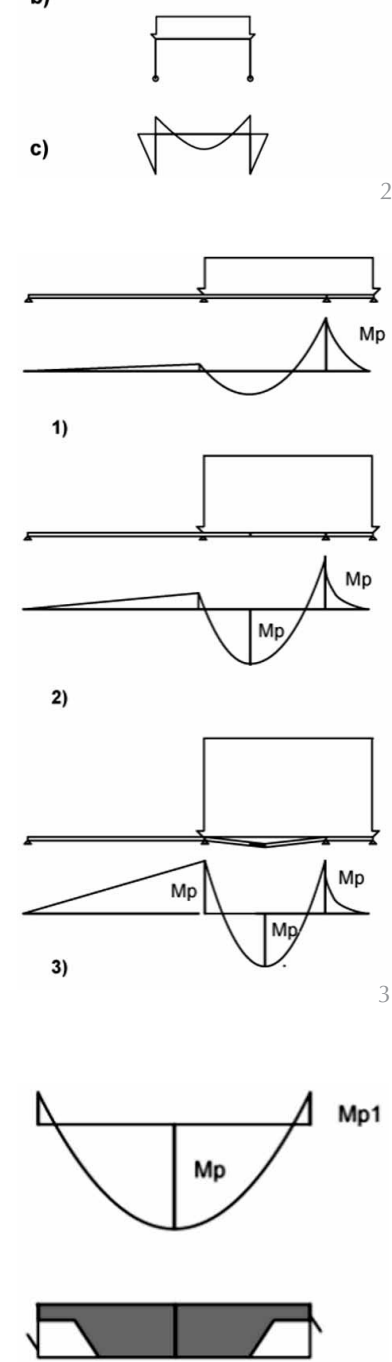

a)
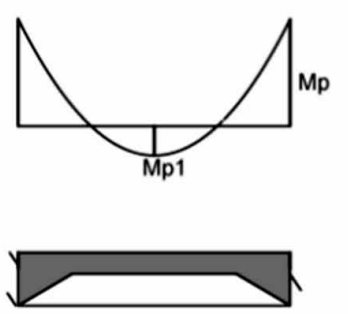

b)

2. Viga con distintos empotramientos elásticos.

3. El proceso de rotura de la estructura a).

4. Configuraciones de equilibrio de una viga empotrada y sus estructuras. 


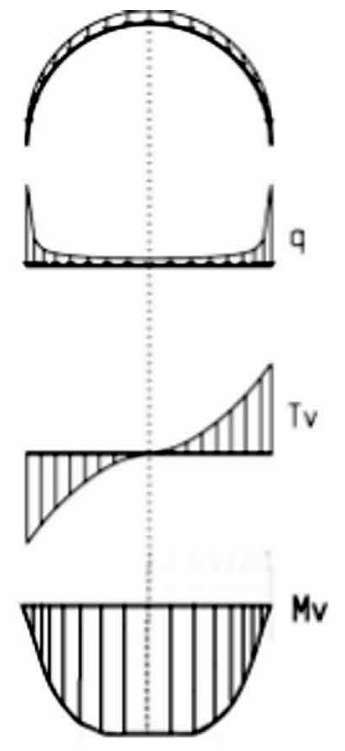

a)

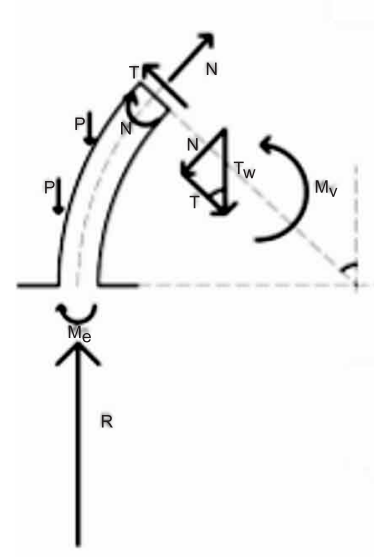

b)
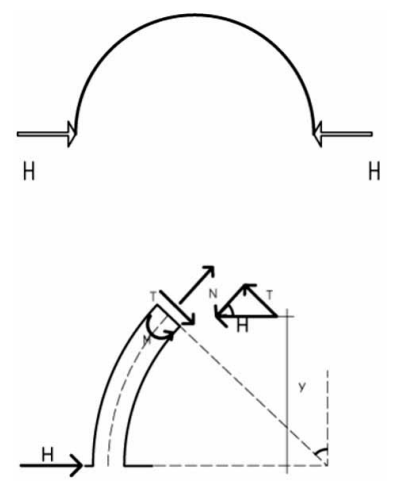

6

5. a) Esfuerzos globales de una viga en arco. b) Esfuerzos en una sección inclinada.

6. Esfuerzos en una sección inclinada debidos al empuje. torsores y, por tanto, trabaja según bandas en dos direcciones.

Sin embargo, quizá la aplicación más conocida de este teorema es el método debido a Heyman para determinar la seguridad de los arcos de fábrica. Antes de entrar a analizar éste método, es conveniente repasar algunas nociones fundamentales sobre la estática de los arcos.

\section{LA ESTÁTICA DE LOS ARCOS, LOS ANTIFUNICULARES Y LA LÍNEA DE EMPUJES}

Como cualquier albañil experimentado sabe, un arco no es cualquier estructura lineal curva, sino sólo una estructura curva en la que los desplazamientos horizontales en los apoyos están de una forma u otra parcial o totalmente impedidos. Para comprenderlo empecemos estudiando una estructura curva en la que no impedimos los desplazamientos horizontales en la base, es decir, una "viga en arco". Si la carga es vertical, al ser las reacciones verticales, serán las mismas que las de la viga horizontal en la que actúan la carga proyectada $q$, y por tanto el momento global Mv y el cortante global Tv, será exactamente el de la viga de igual luz que soporta la carga q (Figura 5 a).

Si la carga es simétrica y hemos impedido los giros, aparecerá en los dos apoyos de la estructura un momento de empotramiento Me, que se restará o sumará a Mv.

Si ahora damos un corte perpendicular a su directriz (Figura 5 b), vemos que si bien el momento $M$ en la sección sería el mismo de la viga proyección: $M=M_{v}+$ $M_{e}$, el cortante $T$ de la sección disminuye al estar la sección inclinada: $T=T_{v} \cos \gamma$ debido a que aparece una compresión que le ayuda a soportarlo: $N=-T_{v} \operatorname{sen} \gamma$.

Supongamos ahora que impedimos el desplazamiento en los apoyos. Desde el punto de vista estático esto implica (Figura 6) la aparición de dos fuerzas horizontales en los apoyos, es decir, dos "empujes" H. Si estudiamos aisladamente el efecto del empuje en una sección inclinada del arco vemos que produce:

Un momento negativo: $M=-H \cdot y$; un cortante de signo contrario a $T_{v} ; T=-H \operatorname{sen} \gamma ; y$ una compresión: $N=-H \cos \gamma$. Si ahora sumamos este efecto al de la carga obtenemos las ecuaciones del arco:

$\left\{\begin{array}{l}M=M_{v}+M_{e}-H \cdot y \\ T=T_{v} \cos \gamma-H \operatorname{sen} \gamma \\ N=-T_{v} \operatorname{sen} \gamma-H \cos \gamma\end{array}\right.$

Vemos que el efecto del empuje es no sólo disminuir el momento y el cortante del arco, sino producir una compresión importante en todas las secciones del arco. Esto explica la existencia de arcos formados con materiales con resistencia prácticamente nula a tracción, como la fábrica: la combinación de un momento flector pequeño con una compresión grande, anula o hace muy pequeñas las tensiones de tracción.

Examinando las ecuaciones del arco, vemos que si suponemos que no hay empotramiento: $M e=0, y$ damos a la directriz " $y$ " del arco la forma del diagrama de momentos dividida por el empuje, que en este caso se comporta como una constante: $y=M_{v} / H$, la inclinación del arco será: $\operatorname{tg} \gamma=\delta y / \delta x=T_{v} / H$, y las ecuaciones del arco se reducen a:

$$
\left\{\begin{array}{l}
M=0 \\
T=0 \\
N=\frac{H}{\cos \gamma}
\end{array}\right.
$$

El arco se transforma en un "antifunicular", es decir, una estructura que solo trabaja a compresión. Tradicionalmente el antifunicular se obtenía a partir de un polígono de fuerzas, aunque actualmente hay tantas razones para usar este polígono para calcular el antifunicular, como para calcular el diagrama de momentos. Su uso más extendido fue, no para encontrar la forma ideal de un arco, sino para dibujar el diagrama de momentos $M$ de un arco a determinada escala. En efecto, si dividimos la primera ecuación de [1] por $\mathrm{H}$, obtenemos:

$M / H=M_{v} / H+M_{e} / H-y$

Es decir, la relación entre el momento y el empuje, es la diferencia entre la directriz del antifunicular desplazada en vertical, y la directriz del arco, Figura 7.

Es por ello que al antifunicular de la carga desplazado $M_{\mathrm{e}} / H$ se le llamó "línea de empujes". Por supuesto el momento $M / H$ aparece con su verdadero signo, es decir, el positivo por encima de la directriz del arco y negativo por debajo. Bastaba pues desplazar el antifunicular a lo largo de la directriz del arco para obtener el diagrama de $M / H$, medido en vertical.

Se calcule el arco de esta forma o de otra, queda sin embargo por resolver el problema básico: obtener el empuje $\mathrm{H}$ y el momento de empotramiento en los apoyos Me. Si el arco no se desplaza en sus apoyos y está empotrado, un razonamiento elemental puede servirnos para determinar la forma del diagrama de momentos: es evidente que (Figura 8) un momento positivo produce desplazamientos hacia fuera (Figura $8 a$ ), mientras que uno negativo produce desplazamientos hacia den- 
tro (Figura 8b). El diagrama de momentos de un arco sin desplazamientos en los apoyos debe ser tal que los momentos negativos y positivos sean aproximadamente de la misma magnitud (Figura 8c), es decir, un diagrama de momentos "compensado".

De hecho, Candela calculaba los arcos de hormigón armado (6) desplazando en vertical el diagrama de momentos de un arco de tres articulaciones -que, como todos sabemos, está estáticamente determinado- hasta que los momentos positivos igualaban en más o en menos a los negativos (Figura $8 \mathrm{~d}$ ). De la misma forma, haciendo que la línea de empujes pase por encima y por debajo de la directriz del arco obtenemos un diagrama de momentos "compensado".

Si suponemos que el material sigue la ley de Hooke, no se fisura y además es capaz de resistir cualquier tipo de solicitación en período elástico, se pueden obtener el empuje y el momento de empotramiento del arco sin más que plantear que en los apoyos los giros y los desplazamientos son nulos. Esto conduce a un diagrama "compensado" con el matiz de que al ser los desplazamientos el producto de los giros por la altura del arco, el diagrama debe ser tal, si el arco es de sección constante, que la suma de los productos de los momentos por las alturas se anule a lo largo del arco y además que las áreas de momentos positivos sean iguales a las de momentos negativos. Esto implica que los momentos en las claves sean ligeramente menores que en los riñones (Figura 9a, pág. 62). Sin embargo, a medida que vamos admitiendo desplazamientos horizontales en los apoyos -cosa casi inevitable en arcos de fábrica- el diagrama elástico empieza a modificarse profundamente. Por lo que acabamos de exponer, para que aparezca un desplazamiento hacia fuera los momentos positivos deben superar a los negativos con lo que el empuje debe disminuir, y los diagramas, a medida que aumentan los desplazamientos (Figura 9b, pág. 62), empiezan a parecerse cada vez más a los de una viga en arco.

Cuando el desplazamiento es el de una viga en arco, el empuje se hace cero y la estructura deja de trabajar como un arco. Por supuesto, esto es cierto para estructuras que siguen la ley de Hooke, y trabajan en período elástico -como arcos metálicos o en celosía- pero muy difícilmente puede serlo para arcos de fábrica. En realidad pueden encontrarse en estructuras históricas, arcos de fábrica con grandes desplazamientos en los apoyos y fuertemente fisurados que aparentemente siguen resistiendo la carga, a pesar de que la teoría elástica predeciría su colapso. Como demostró Heyman (7) para comprobar un arco de fábrica es casi inevitable recurrir a los teoremas de rotura, como vemos a continuación.

\section{CÁlCUlO A ROTURA de ARCOS DE FÁBRICA}

En primer lugar, debemos comprobar si una estructura sin resistencia a tracción -y esto es lo que prácticamente ocurre en una estructura de fábrica cuando los esfuerzos son perpendiculares a las juntas- puede resistir, aunque esté fisurada, un momento flector $M$, con tal de que simultáneamente actúe un normal N. Efectivamente, si la sección está sobredimensionada a compresión, es decir, tiene un canto $h$ mayor del canto $h_{0}$ que necesitaría estrictamente para resistir el normal, las tensiones de compresión pueden acumularse en la zona no fisurada de forma que produzcan una resultante $\mathrm{N}$ que equilibre el normal (Figura 10a, pág. 62). Si la sección es rectangular de lado b y suponemos -como en el hormigón- una distribución de tensiones constante, podemos obtener de forma elemental la longitud $h_{0}$ de la zona no fisurada, y el momento $M$ que puede resistir la sección (Figura 10b, pág. 62). Por equilibrio de fuerzas verticales:

$N=f \cdot b \cdot h_{0} ;$ y $h_{0}=N / f \cdot b$

si llamamos f a la tensión de la fábrica, incluyendo en su valor los coeficientes de seguridad de las cargas y del material. La resultante de las $\mathrm{f}$ produce con el normal $\mathrm{N}$ un par de fuerzas, que puede equilibrar el momento:

$M=N \cdot$ e, donde el brazo de palanca e es: $e=h / 2-h_{0} / 2$, queda:

$M=N \frac{h-h_{0}}{2}$

Vemos que una sección fisurada puede resistir un momento $M$, con tal de que esté comprimida con un normal N, y esté sobredimensionada a compresión.

Si despejamos h, de [4], podemos obtener la expresión de lo que podríamos llamar el "criterio de Heyman":

$h \geq h_{0}+2 M / N$

"Para que una estructura de sección rectangular resista un momento $M$ sin tracciones, su canto debe sumar al menos su canto estricto $h_{0}$ y dos veces su excentricidad M/N". Gráficamente, podemos dimensionar la sección (Figura 10c, pág. 62) colocando a cada lado del eje dos excentricidades $M / N$, y dos medios cantos estrictos $h_{0} / 2$. Vemos que en realidad, el valor determinante para la resistencia de la fábrica no es el diagrama de
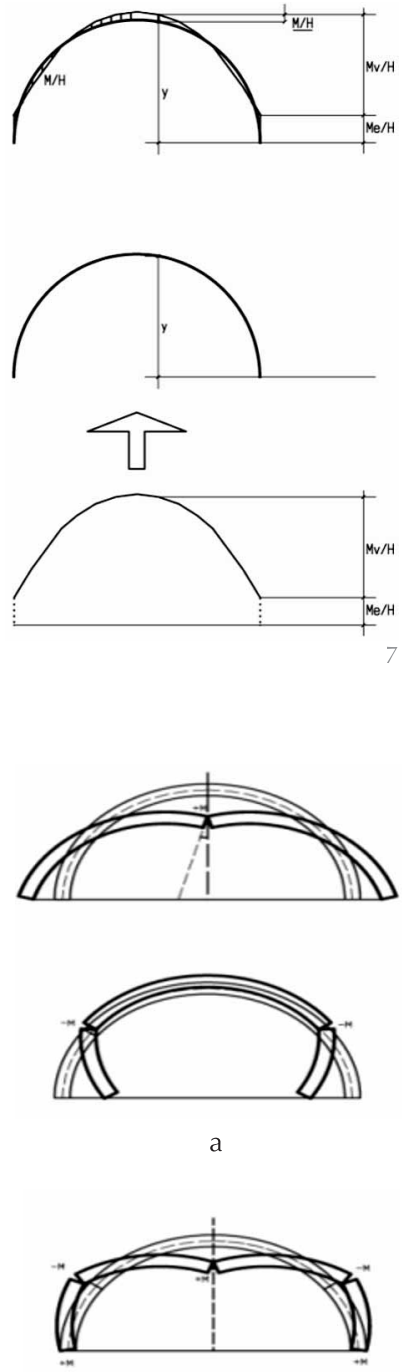

b

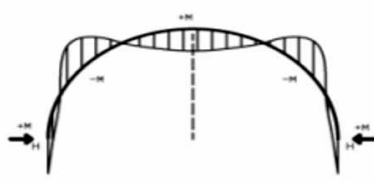

C

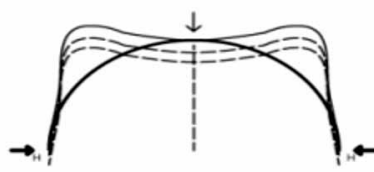

d

Relación entre el antifunicular, la ordenada del arco y, y el momento $M$.

8. a) Relación entre momento positivo y desplazamiento hacia afuera. b) Relación entre momento negativo y despalazamiento hacia dentro. c) Momentos y emplazamiento nulo. c) Método de Candela. 

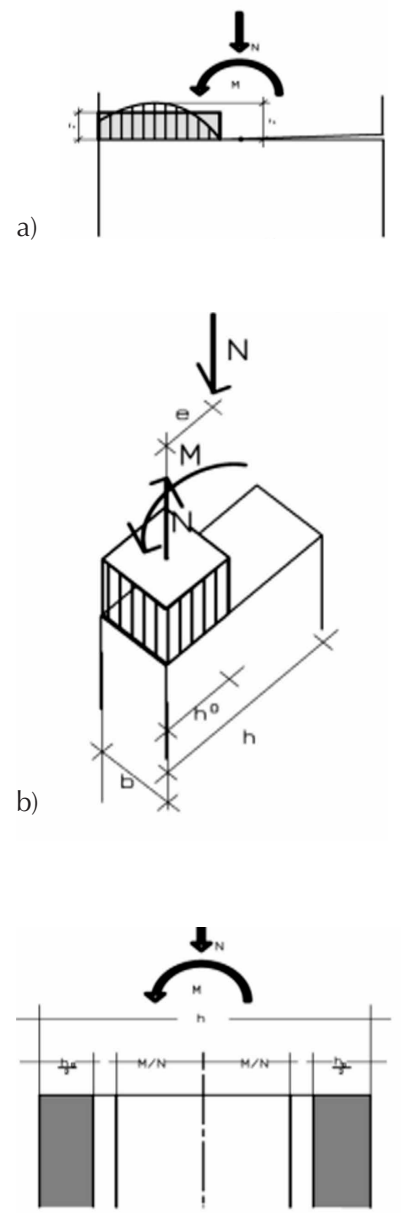

C)

9. a) Momentos de un arco empotrado sin desplazamientos en los apoyos. b) Momentos de un arco empotrado con desplazamientos en los apoyos.

10. a) Tensiones reales y tensiones simplificadas en una sección fisurada de fábrica. b) Equilibrio en una sección de fábrica. c) Criterio de Heyman.

(*) En realidad Heyman despreció $h_{o}$ suponiendo que era muy pequeño basándose en el siguiente razonamiento: si en la ecuación [4] introducimos el valor $h_{0}$ de [2] obtenemos: $M$ $=N h / 2\left(1-N / N_{u}\right)$, donde $N_{u}$ es la capacidad máxima de la sección para resistir una compresión: $N_{u}=f \cdot b \cdot h$. Si dibujamos el diagrama $M-N$, obtenemos el de la figura. Vemos que la capacidad máxima de la sección para resistir un momento es para $h_{0}=h / 2, N=N_{u} / 2, y$ que cuando $h=h_{0}, N=N_{u}$ la sección se agota a compresión

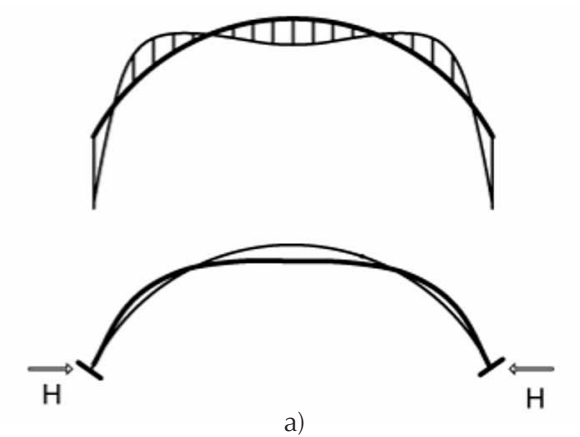

ma de la unicidad de la solución, este desplazamiento es una condición de contorno elástica en servicio que no influye ni en la configuración ni en los esfuerzos de rotura. La única condición es, como vimos, que puedan formarse rótulas plásticas en la estructura que bloqueen el momento cuando éste alcance su valor último.

Parece posible que cuando la línea de presiones se hace tangente al límite de la sección central, es decir se fisura con el diagrama de tensiones de la clave del arco de la Figura $11 b$, se comporte como tal rótula plástica: al mantenerse el normal, continúa pudiendo contrarrestar el momento, aunque aumente el ancho de la fisura. Esto ha sido confirmado por los experimentos llevados a cabo en la U.P.C., y otros centros ${ }^{(* * *)}$. El proceso de rotura de un arco que en servicio se le han desplazado los apoyos a cada lado un valor u, podría describirse de la forma que se indica en la Figura 12a, pág. 64.

Supongamos que empieza el desplazamiento, si el arco continúa trabajando en servicio, los momentos positivos empezarán a aumentar, disminuyendo los negativos y el empuje como en la Figura 9, hasta que en la clave el momento alcance su valor último, o, lo que es lo mismo, la línea de presiones se haga tangente al límite de la sección central en ese punto. A partir de ahí el momento en la clave se bloquea, y el diagrama de momentos, el de normales y por tanto la línea de presiones se mantienen iguales, aunque continuemos desplazando los apoyos. Si queremos averiguar su forma de rotura, podemos aumentar la carga progresivamente.

Cuando en los arranques la línea de presiones se haga tangente a la sección central -que en el proceso habrá ido disminuyendo progresivamente al aumentar $h_{0}-$ se formarán dos rótulas plásticas y, por tanto, esfuerzos y línea de presiones se bloquearán en esos puntos.

Finalmente si continuamos aumentando la carga, se formarán dos rótulas en los riñones, el arco se transformará en un mecanismo y la estructura colapsará (Figura 12a). El diagrama de momentos en rotura será aquél que haga la línea de presiones tangente al límite de la sección central en cinco puntos (Figura 12b y c) y será, por supuesto, el mismo que tendría el arco si no se hubiesen desplazado sus apoyos en servicio, o hubieran sufrido estos un asentamiento diferencial. Si lo comparamos con el diagrama de servicio con los apoyos sin desplazar (el de la Figura 11a), vemos que son muy semejantes. Ambos "compensados" y se diferencian en el valor relativo del momento de empotramiento, menor para el diagrama en rotura. 
Podríamos realizar un cálculo a rotura del arco en sentido estricto calculando los diagramas de momentos en rotura -por ejemplo utilizar el diagrama de momentos de Candela y corregirlo aumentando o disminuyendo el valor del empuje- y dimensionando el arco para que, cuando la carga aumente el coeficiente de seguridad, $\mathrm{h}_{0}$ aumente de tal forma que la sección central quede tangente a la línea de presiones en cinco puntos.

Sin embargo, Heyman (7), demostró que en realidad, si lo que queremos es asegurarnos de que el arco es capaz de resistir unas cargas determinadas, puede tomarse como válido cualquier diagrama de momentos con tal de que corresponda a una línea de presiones contenida dentro de la sección central. Efectivamente, si aplicamos el teorema del límite inferior, cualquier diagrama de momentos y normales que corresponda al equilibrio de la carga de rotura supuesta y cuya línea de presiones esté dentro de la sección central sin ser tangente a su límite en los puntos en que aparecen las rótulas, es una configuración de equilibrio no de rotura y, por tanto, la carga de rotura del arco debe ser mayor que la carga de rotura supuesta.

Como vimos, no todos los máximos del diagrama deben ser mayores que el de rotura, por lo que no debería utilizarse ese diagrama para dimensionar el arco, pero aun así, las diferencias entre diagramas "compensados" serían tan pequeñas que nunca se cometería un gran error.

\section{LA LÍNEA DE EMPUJES, EL MÉTODO DE LA ESTRUCTURA INTERNA Y LOS ANTIFUNICULARES}

Los métodos del límite inferior aplicados a arcos han adquirido tanta popularidad que es difícil encontrar un estudio sobre arcos de fábrica en los que no se mencionen o se utilicen. Sin embargo, casi nunca se aplica correctamente: en lugar de utilizar la línea de presiones se utiliza la línea de empujes -la directriz de uno de los antifuniculares de la carga, como vimos- y se considera seguro un arco si se encuentra una línea de empujes que esté contenida dentro de la sección del arco. Como vimos en el apartado 4, la distancia vertical entre la directriz del arco y una línea de empujes que corresponde a un empuje $\mathrm{H}$ es la relación entre momento y empuje $M / H$, no la relación entre momento y normal $M / N$ medida en dirección perpendicular al arco.

Podría aducirse que como prácticamente siempre -al menos si el arco está sometido a cargas verticales- el normal es siempre mayor que el empuje, utilizar la línea de
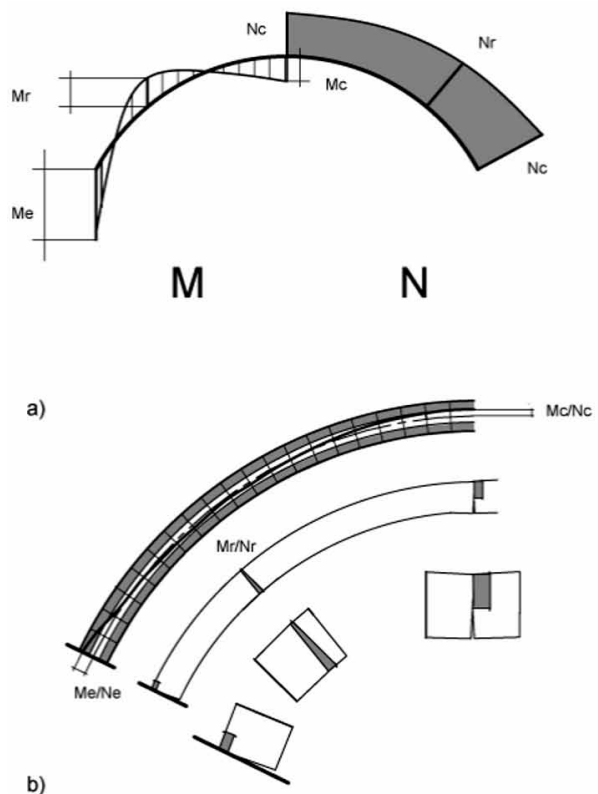

empujes está del lado de la seguridad. Sin embargo, en tal caso, habría que medir esta distancia, no en vertical, sino en dirección perpendicular a la directriz del arco, girando $\mathrm{M} / \mathrm{H}$ en esa dirección y comprobando si se encuentra dentro de la sección central del arco. Quizá la razón de la popularidad de esta aplicación incorrecta, es que se piensa que si existe una línea cuyos esfuerzos están en equilibrio con la carga dentro del arco, de alguna manera éste debe ser capaz de resistir la carga. Lo que intuitivamente se adivina con este razonamiento, es uno de los métodos derivados del segundo teorema, lo que podríamos llamar "método de la estructura interna". Veamos en qué consiste.

Evidentemente, una de las configuraciones de equilibrio - no de rotura- a las que se refiere el segundo teorema podría ser, simplemente, una estructura que estuviese incluida dentro de la estructura real. Aquí el segundo teorema se hace casi obvio: si podemos definir dentro de la estructura real otra estructura -por ejemplo, las vigas acarteladas de la Figura 4- que resista la carga de rotura de cálculo, es evidente que la estructura real -que contiene más material- debe ser capaz de resistir una carga mayor.

Como en todos los métodos derivados del segundo teorema, esto no implica que en determinados puntos de la estructura interna no puedan aparecer esfuerzos menores que los de rotura de la estructura real. Quizá la aplicación más popular de este método es el método de la celosía interna aplicado a vigas de hormigón introducido por Mohr y Ritter en el S. XIX, y desarrollado casi exhaustivamente por Schlaigh y otros como el y es incapaz de resistir un momento. Si $N_{u}$ es muy grande en relación a $N, M=N h / 2$, y se puede despreciar $h_{0}$. Heyman estableció que esto ocurre siempre porque el coeficiente global de seguridad de la fábrica suele tener valores de alrededor de 10. Sin embargo, puede objetarse que si el coeficiente de seguridad es muy alto es porque existe una fuerte probabilidad de que la tensión de rotura sea mucho más baja de lo previsto $y$, además, los constructores de las estructuras históricas nada sabían de coeficientes de seguridad, como prueban las secciones aplastadas parcialmente a compresión que puede encontrar cualquiera que se dedique a la restauración.

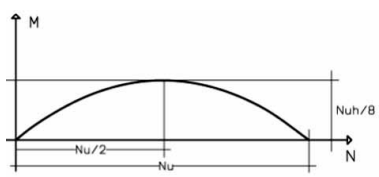

DIAGRAMA N-M

${ }^{(* *)}$ Lo que podríamos llamar "criterio de Young", ya que fue descrito por primera vez por Young en 1802, se basa en lo siguiente: si en una sección rectangular de canto $h y$ ancho $b$, sometida a flexo-compresión sumamos los diagramas elásticos, o en servicio, obtenemos que la tensión mínima es: $\sigma_{\min }$ $=-N / b h+6 M / b h^{2}$, si queremos que $\sigma_{\min }<0$, es decir, que sea una compresión._O $>-N$ / $b h+6 M / b h_{2}$ y por tanto: $M / \underline{N}$ $<h / 6$, es decir la excentricidad $M / N$ debe encontrarse dentro del "tercio medio" de la sección.

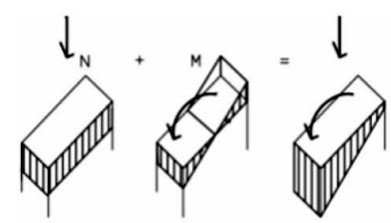

CRITERIO DE YOUNG

${ }^{(* *)}$ Pueden encontrarse los resultados de los ensayos sobre arcos de fábrica llevados a cabo en la U.P.C. bajo la dirección de P. Roca, y de otros llevados

11. a) Momentos (izda.) y Normales (drcha.) de un arco empotrado, b) Línea de presiones del mismo arco. 
a cabo en distintos laboratorios en la Tesina de la U.P.C.: Marco Ibáñez, Germán. "Ensayo hasta Rotura de un Puente Arco de Obra de Fábrica Construido en Laboratorio". Tesina U.P.C. bajo la dirección de P. Roca Fabregat. Barcelona, Noviembre, 2002.
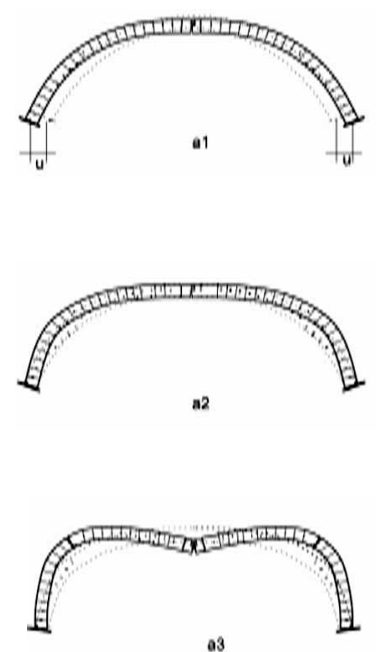

a)

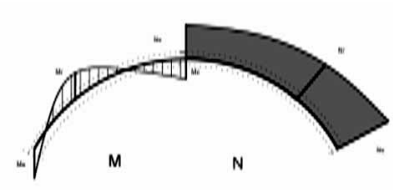

b)

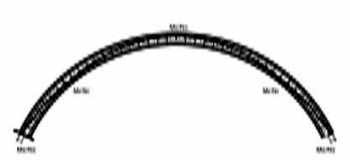

c)

12

12. a) Proceso de rotura de un arco con un desplazamiento inicial $u$. a1. Formación de la rótula en clave. a2. Formación de rótulas en los arranques. a3). Formación de rótulas en los riñones. b) Diagrama de momentos (izda.) y Normales (drcha.) en rotura.c) Línea de presiones en rotura.

13. Estructura interna de una viga pared y sus isostáticas de (5).

14. Línea de presiones (izda.) y antifunicular (drcha.) de un arco apuntado.
13

"método de la bielas y los tirantes" (5). En esencia, se analiza una viga de hormigón a partir de una celosía contenida en ella, cuyas barras a tracción son las armaduras, y cuyas barras a compresión son parte del propio hormigón.

Schlaigh, y los demás autores, son por supuesto muy conscientes de que los esfuerzos internos de la estructura real pueden ser muy distintos de los de la celosía interna y dedican todos sus esfuerzos a adaptar la forma de la celosía a las direcciones principales de la estructura real. Así, en la Figura 13, se opta por una celosía con el canto mitad del de una viga pared, pues de otra forma los esfuerzos de la celosía interna podrían duplicar los esfuerzos de la estructura real para la misma carga.

En el caso de un arco, podríamos concebir la estructura interna como uno de los antifuniculares de la carga que estuviese contenido dentro del arco, y cuya directriz es efectivamente la "línea de empujes".

Sin embargo, la aplicación del método implica que el antifunicular sea una estructura, es decir, debe tener la sección necesaria para

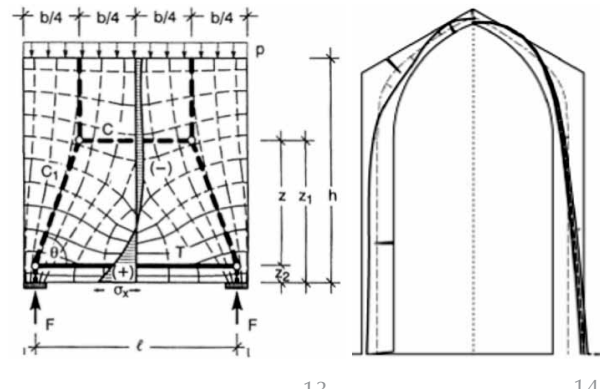

resistir las tensiones de compresión producila forma de la línea de empujes como guía para determinar los puntos en que el arco sufre los máximos esfuerzos.

En la Figura 14, el antifunicular tendría un espesor pequeño en la clave, pero no ocurriría lo mismo en los arranques, en los que dejaría de ser una "estructura interna", aunque la línea de empujes esté dentro del arco, y no daría pistas sobre el máximo de esfuerzos que aparece en los riñones, como indica la línea de presiones.

\section{CONCLUSIONES}

Podemos concluir que si bien es posible, e incluso recomendable, utilizar los teoremas de rotura para comprobar arcos de fábrica:

1) El verdadero criterio es que la línea de presiones se encuentre dentro de la sección central del arco, para la carga de rotura de cálculo y no que la línea de empujes esté dentro del arco.

2) Se puede utilizar la línea de empujes como directriz de un antifunicular de la carga, pero en este caso se trata de encontrar una estructura interna, con sus espesores, que resista la carga.

3) En tal caso se podrá afirmar que la carga de rotura real es mayor que la del antifunicular, pero de ninguna manera la línea de empujes se puede tomar como guía de los puntos en los que el arco sufra fisuraciones o se estén formando rótulas plásticas. das por la carga, y además no puede tomarse

\section{REFERENCIAS}

(1) Johansen, K.W.: "Yield-Line Theory" Cement and Concrete Association. Londres, 1962.

(2) Heyman, J.: "Análisis de Estructuras" Instituto Juan de Herrera. Madrid 2004. Traducción de:" Structural Analysis, a Historical Approach" Cambridge University Press, 1998.

(3) Kurrer, E.: "The History of the Theory of Structures" Erns and Sohn. Berlin, 2008.

(4) Nielsen, M.P.: "Limit Analysis and Concrete Plasticity" Prentice Hall. New Jersey, 1984.

(5) Schlaigh J. y otros.: "Towards a Consistent Design of Structural Concrete". P.C.I. Journal. Vol. 32, n. 3 Mayo-Junio 1987

(6) Faber, C.: "Las Estructuras de Candela" C.E.C.S.A. Méjico, 1970.

(7) Heyman, J.: "The Stone Skeleton". International Journal of Solids and Structures. Vol. 2, pp. 249-279. 1966.

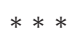

